\title{
MAGNETIC FIELDS OF RADIO GALAXIES IN ABELL CLUSTERS 115 AND 568
}

\author{
L. Gregorini ${ }^{1,2}$, M. Bondi ${ }^{2}$, L. Feretti ${ }^{1,2}$ G. Giovannini ${ }^{1,2}$, C.P. O'Dea ${ }^{3}$ \\ 1 Dipartimento di Astronomia, Università di Bologna, Italy \\ 2 Istituto di Radioastronomia del CNR, Bologna, Italy \\ 3 Netherland Foundation for Radio Astronomy, Dwingeloo, \\ The Netherlands
}

\section{Abell 115}

Abell 115 is an intermediate-redshift cluster $(z=0.1971)$ well studied at different wavelengths (see Giovannini et al., 1987 and references therein).

A Narrow Angle Tail radio galaxy is located at about $480 \mathrm{kpc}\left(\mathrm{H}_{0}=100 \mathrm{~km}\right.$ $\mathrm{s}^{-1} \mathrm{Mpc}^{-1}$ throughout this paper) from the center of the cluster. The radio source is associated with a cluster member whose deprojected velocity, with respect to the mean cluster velocity, is about $2400 \mathrm{~km} \mathrm{~s}^{-1}$.

The radio characteristics of the NAT radio source are: a core coincident with the optical galaxy, an asymmetric jet with a bending at $6 \mathrm{kpc}$ from the nucleus, a tail structure resolved in two streams of emission, and a low brightness feature which bends at $60 \mathrm{kpc}$ in direction opposite to the $\mathrm{X}$-ray emission centered on $3 \mathrm{C}$ 28. The core, the jet and the counterjet are not polarized. The southern knot is more polarized than the northern one $(11 \%$ against $9 \%$ at $1.5 \mathrm{GHz}, 22 \%$ against $17 \%$ at $4.9 \mathrm{GHz}$ ), and the magnetic field becomes circumferential towards the knot edges.

We have computed the equipartition magnetic field for various components of the source (Figure 1). A detailed discussion of this source is given in Gregorini and Bondi (1989).

\section{Abell 568}

Abell 568 is a closer cluster $(\mathrm{z}=0.0751)$ characterized by a small group of galaxies located in its center within a linear distance of about $65 \mathrm{kpc}$. Analysis of the radio data is in progress; only preliminary results are given.

The peculiar radio emission of this group consists of two radio galaxies (Figure 2). The western radio source is associated with the brightest member of the small group $(z=0.0790)$. The eastern radio galaxy is a Wide Angle Tail with an opening angle of about $90^{\circ}$ and is associated with a galaxy $(z=0.0737)$ which shows an elongated symmetric feature at the same position angle of the radio jets.

The two radio galaxies belong to Fanaroff and Riley Class I and both show two symmetric jets. The jets in the eastern radio source are polarized with magnetic field perpendicular to the axis (neglecting Faraday rotation effects); the field 
becomes tangential on the edges of the radio source. In the western radio source only the tails are polarized. Computed values of equipartition magnetic field are superimposed in figure 2.

The distorted radio morphology and the physical proximity of galaxies suggest that we may be witnessing the merging of two or more galaxies in the cluster center.

\section{References}

Giovannini, G., Feretti, L., Gregorini, L. (1987) Astron. Astrophys. Suppl. Ser. 69, 171

Gregorini, L. , Bondi, M. (1989) Astron. Astrophys., in press

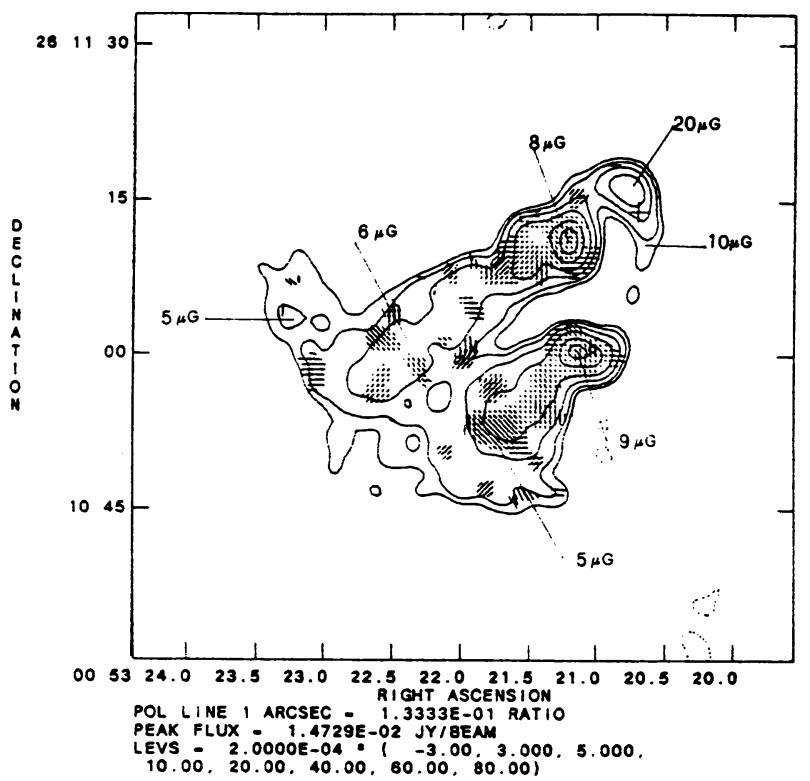

Fig. 1 (left): A 115

VLA map at $1.5 \mathrm{GHz}$

(FWHM beam of $2^{\prime \prime} .5 \times$ $\left.2^{\prime \prime} .5\right)$

Fig. 2 (below): A 568

VLA map at $4.9 \mathrm{GHZ}$ (FWHM beam of $2^{\prime \prime} .1 \times$ $1^{\prime \prime} .4\left(64^{\circ}\right)$ )

In both maps $\mathrm{E}$ vectors are proportional to fractional polarization

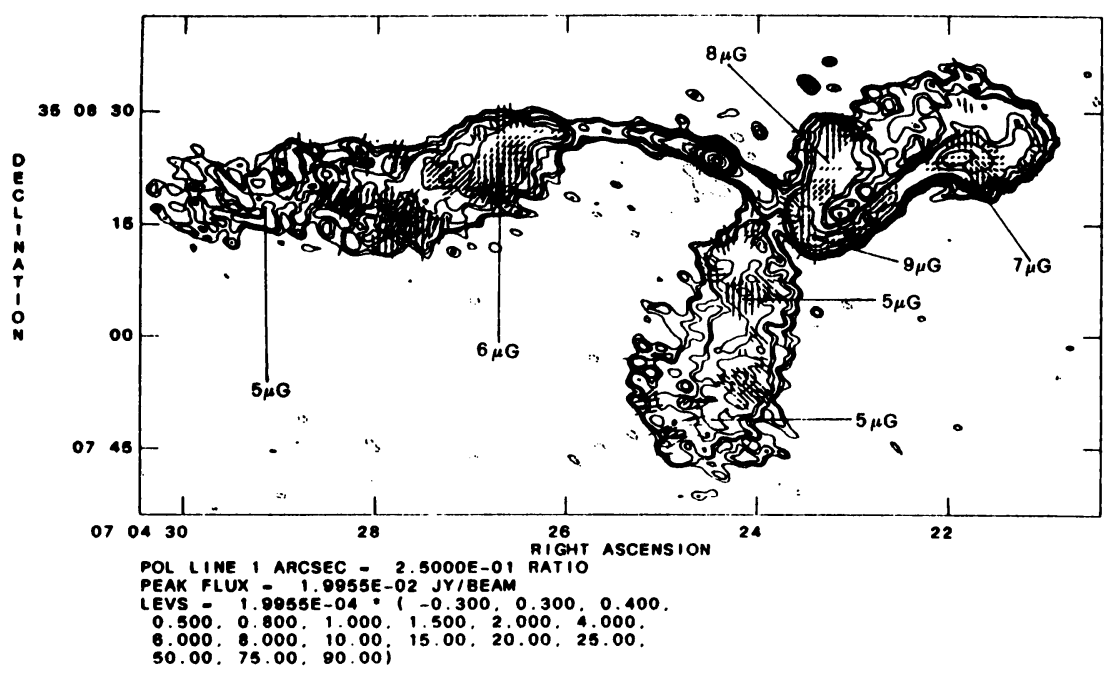

